\title{
Thromboembolic complications after treatment with monoclonal antibody against CD40 ligand
}

To the editor-Exciting results reporting that blockade of the CD40/CD40 ligand pathway by monoclonal antibody against CD40 ligand led to long-term acceptance of renal allografts in monkeys were recently reported in Nature Medicine $^{1}$ and elsewhere ${ }^{2}$. Our own group has previously reported that mixed chimerism and renal allograft tolerance are achieved in monkeys conditioned with a multi-modality peritransplant regimen and without longterm maintenance immunosuppression ${ }^{3,4}$. In an effort to simplify this therapeutic regimen, we tested a monoclonal antibody against CD40 ligand (American Type Culture Collection catalog number $5 \mathrm{C} 8.33$ ) in this protocol. In the first animals treated, we encountered an unusually high incidence of thromboembolic complications, which we thought worthy of reporting.

Using our standard regimen (nonmyeloablative total body irradiation, thymic irradiation, anti-thymocyte globulin, donor bone marrow infusion and a 1-month course of cyclosporine), we found no thromboembolic complications in more than 50 animals. In contrast, after adding $20 \mathrm{mg} / \mathrm{kg}$ of monoclonal antibody against CD40 ligand to the regimen on days 0 and 2, we observed four thromboembolic complications in nine recipients. These included two renal artery thromboses, one renal vein thrombosis and one superior mesenteric artery thrombosis. In subsequent animals, the addition of 100 units/kg heparin immediately before the antibody (days 0 and 2) reduced the incidence of thrombotic complications to just two (one renal artery, one renal vein) in ten recipients. We then tried additional heparin treatments (100 units/kg, days $0,1,2,3)$ preceded by vigorous postoperative hydration. None of the five animals tested after this change have developed thrombotic complications. These observations may be relevant to ongoing clinical trials of monoclonal antibody against CD40 ligand (humanized version of 5C8) in which some thromboembolic complications have been reported (Vincent, J. Biogen News www.prnewswire.com, 11/2/99).

CD40 ligand was originally identified on activated $\mathrm{CD} 4^{+} \mathrm{T}$ cells ${ }^{5}$, later on stimulated mast cells and basophils ${ }^{6}$ and most recently on activated platelets in vitro and in vivo on platelets in the process of thrombus formation ${ }^{7}$. CD40 is constitutively expressed on the vascular endothelium of various organs, and its ligation can upregulate adhesion molecules such as E-selectin, VCAM-1 and ICAM-1. Ligation through CD40 has also been reported to upregulate tissue factor expression on endothelial cells ${ }^{8}$. CD40 ligand expression has also been seen in vivo on activated platelets by examining fresh thrombi formed during vessel injury. In all fresh thrombi analyzed, CD40 ligand was expressed on a large proportion of platelets in areas in which densely packed platelets had not yet formed an amorphous mass and on platelets directly adhering to the vessel endothelium. These results may be relevant to the mechanism of a possible increased risk factor for thromboembolism induced by monoclonal antibody against CD40 ligand. Our results indicate that the administration of heparin in conjunction with monoclonal antibody against CD40 ligand can reduce the incidence of thromboembolic complications.

TAtsuo KaWAi, David ANDrEWs, Robert B. Colvin, David H. SaChs \& A. Benedict Cosimi

Departments of Surgery and Pathology

Harvard Medical School

Massachusetts General Hospital

Boston, Massachusetts, USA

Kirk and Harlan reply-Kawai and colleagues report a considerable number of thromboembolic events in monkeys after administration of a mouse monoclonal antibody against human CD154. This group's extensive experience with the monkey kidney allograft model, coupled with their unprecedented run of thrombotic complications, does suggest that something is awry, perhaps that the antibody preparation used in their experiments is prothrombotic. Indeed, a link between CD154:CD40 and coagulation makes great teleological sense. Nevertheless, what makes sense and what is fact are often very different. However appealing, given the reported presence of CD154 on platelets and endothelia, we believe it premature to definitively conclude that the results discussed in this letter are epitope-specific.

Thromboembolic complications have been found after the initial use of many antibodies in humans and monkeys, and have largely disappeared with the use of refined antibody purification processes. Biogen's early clinical trials have been marred by thromboembolic events, indicating that humanized antibody against human CD154 induces thrombosis. These complications have been publicly disclosed and the trials have been halted pending additional pre-clinical evaluation. However, trials (admittedly different in design) using an antibody against human CD154 produced by IDEC Pharmaceuticals have proceeded so far without reported thromboembolic events.

Agents interfering with the CD154: CD40 pathway obviously powerfully influence the immune system and will no doubt have collateral effects. Potential effects, such as the ones presented here, must be scrutinized methodically and then corroborated by rigorous prospective investigation. Only then can important clues derived from anecdotes be accepted as fact.

Allan D. KirK

DAVID M. HARLAN

1. Kirk, A.D. et al. Treatment with humanized monoclonal antibody against CD154 prevents acute renal allograft rejection in nonhuman primates. Nature Med. 5, 686-693 (1999).

2. Kenyon, N.S. et al. Long-term survival and function of intrahepatic islet allografts in rhesus monkeys treated with humanized anti-CD154. Proc. Natl. Acad. Sci. USA 96, 8132-8137(1999).

3. Kawai, T. et al. Mixed allogeneic chimerism and renal allograft tolerance in cynomolgus monkeys. Transplantation 59, 256-62(1995).

4. Kimikawa, M. et al. Modifications of the conditioning regimen for achieving mixed chimerism and donor-specific tolerance in cynomolgus monkeys. Transplantation 64, 709-716(1997).

5. Armitage, R. et al. Molecular and biological characterization of a murine ligand for CD40. Nature 357 , 80-82(1992)

6. Gauchat, J.F. et al. Introduction of human IgE synthesis in B cells by mast cells and basophils. Nature 365, 340-343(1993)

7. Henn, V. et al. CD40 ligand on activated platelets triggers an inflammatory reaction of endothelial cells. Nature 391, 591-594(1998).

8. Slupsky, J.R. et al. Activated platelets induce tissue factor expression on human umbilical vein endothelial cells by ligation of CD40. Thromb. Haemost. 80, 1008-1014(1998) 